\title{
The influence of higher-order mode shapes for reduced-order models of electrostatically actuated microbeams
}

\author{
Stefanie Gutschmidt* \\ Department of Mechanical Engineering \\ University of Canterbury \\ Private Bag 4800, Christchurch 8140, New Zealand
}

\begin{abstract}
Reduced-order models for micro-electromechanical structures possess several attractive features when compared to computational approaches using e.g. finite element packages. However, also within the business of reduced-order modeling there are different approaches which yield different results. The efficiency of such approaches has to be judged according to, first, the purposes and aims of the model and, second, according to computational expenses and modeling efforts. This paper deals specifically with the frequently asked question of how many modes have to be considered in the discretization procedure to ensure an efficient reduced-order model. A consistent nonlinear continuum model is employed to describe a doubly-clamped microbeam subject to two cases of electromechanical actuation. The analysis, confined to the static behavior, concentrates on two discretization techniques and addresses the differences between the final reduced-order models, accordingly. The results show significant differences with respect to the number of implemented linear-undamped mode shape functions which are used as
\end{abstract}

*e-mail:stef anie.gutschmidt@canterbury.ac.nz 
basis functions in the approximation procedure. This is demonstrated for the two mentioned distinct excitation schemes of the doubly-clamped microbeam. The purposes of this paper are twofold. First, it draws attention to the differences between reduced-order models which have been discretized one way or the other according to investigation goals and purposes. Second, it serves as a guideline for future MEMS/NEMS modeling by elaborating the advantages and disadvantages of both techniques.

\section{Introduction}

Modern treatment of differential equations relies heavily on approximation methods to solve problems of practical interest in macro engineering and even more so when it comes to micro or nano systems. Foremost among the methods used and developed for micro- and nano electromechanical systems (MEMS/NEMS) $[1,2]$ is the finite-element method. Prior to the finite-element method and also to reduced-order models in general is an approximation technique called method of weighted residuals, of which a particular subclass is the GALERKIN method or GALERKIN discretization.

The broad field of research on MEMS and NEMS is addressed by various approaches. The present work aims especially at people whose work put the emphasis on the modeling of such devices and analyzing their behavior $[2,3,4,5,6,7]$. The following summarizes selected contributions in the field of macromodeling for microbeam systems which serve to analyze the subject matter and point to the motivation and objectives of this work. All continuum-based modeling of MEMS begins with an initial boundary value problem formulation including a justification of the assumptions made with respect to the aim of the model. 
An efficient model for the description of the nonlinear static (and dynamic) behavior of a microbeam structure accounts for linear and nonlinear restoring forces (large deflections) [3, 4], visco-eleastic structural and nonlinear squeeze-film damping [6], and the nonlinear electromechanical coupling force. The electro-mechanical coupling force is inversely proportional to the square of the distance between the structure and actuating electrode [8], assuring the parallel capacitor model. An extended review on reduced-order or macro models for MEMS was written by NAYFEH et al. [9]. After introducing non-dimensional quantities (in the procedure of modeling) a reduced-order model, commonly based on the GALERKIN decomposition with undamped linear eigenmodes as base functions, is constructed $[2,9]$. For numerical analysis and comparative investigations to experiments this provides a satisfying tool, which possesses numerous advantages with respect to other approaches $[4,9]$. However, for a continued analytical description of the system's response, the integral of the electromechanical forcing term has to be solved in closed form. Several suggestions have been proposed in literature to deal with this problem. The first and aforementioned approach is to solve the integral actuation term numerically [3] and to set further analytical investigations aside. Another, but "quick and dirty", solution is to expand this term into a TAYLOR series. Numerous examples are available in literature which report a poor accuracy of this approach, even when including multiple higher order terms [4, 9]. Gutschmidt and Gottlieb [7], in their studies on microbeam arrays, bypassed this problem by introducing the actuation only at mid-span of each microbeam. Their emphasis was laid on a pure phenomena and dynamical behavior study, for which radically shortened electrodes served the aims and interests. A new method was introduced by Younis et al. [4], in which the equation of motion was multiplied by the denominator of the electrostatic force before applying the discretization technique. The 
efficiency of all four approaches has to be judged according to, first, the purposes and aims of the model and second, according to computational expenses and modeling efforts. This paper deals specifically with the frequently asked question of how many modes have to be considered in the discretization procedure to ensure an efficient reduced-order model. Based on Younis et al. [4] the number counts the first five symmetric linear undamped modes to be used as basis function. Their work is frequently quoted whenever a justification on the number of modes is sought. The present work shows that the answer to the question on the number of modes significantly depends on the performed discretization technique. Thus, the answer of "five modes" is not a general answer for reduced-order microbeam modeling. Furthermore, one outcome of this work reminds the reader that GALERKIN's decomposition is but a subclass of the method of weighted residuals. As known from the macro system modeling and especially the linear eigenvalue problem, the GALERKIN discretization yields best results due to making use of orthogonal relationships between shape functions.

\section{The method of weighted residuals for the doubly- clamped microbeam}

The vastly used terminology of method of weighted residuals and Galerkin method makes it necessary to begin this section with a description of the meaning of each expression. At this, the author adopts the meanings from the reference [10].

Method of weighted residuals (MWR) "The method of weighted residuals [...] encompasses several methods (collocation, GALERKIn, integral, etc.) ..." (p.4). "The un- 
known solution is expanded in a set of trial functions, which are specified, but with adjustable constants (or functions), which are chosen to give the best solution to the differential equation." (p.7+8)

Galerkin method (GM) "In this method the weighting functions are chosen to be the trial functions, ..." (p.10).

Thus, throughout this manuscript the method of weighted residuals (henceforth abbreviated as MWR) refers to the technique using arbitrary weighting functions while the Galerkin method (henceforth GM) refers to the same technique but using a specific set of trial functions as weighting functions, and thus forming a subclass of the former method.

\subsection{Formulation of the boundary value problem}

The subject is a doubly-clamped microbeam (also known as microbridge) with its dimensions length, width and thickness, considered under two electrode configurations. All quantities in this paper recall the commonly accepted notations for material parameters and dimensions. A typical sketch of the microbeam system for the one-sided electrode configuration is shown in Fig. 1. The first electrode configuration (henceforth referred to as case I) has a full-length

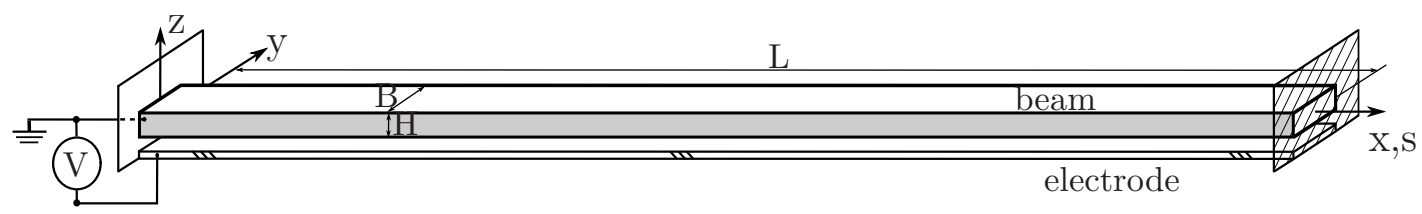

Figure 1: Definition sketch of the clamped-clamped microbeam system for the one-sided electrode configuration. 
electrode on only one side of the beam while the second configuration (henceforth referred to as case II) has full-length electrodes on both sides. The microbeam is made of an elastic material and electrostatically actuated by the electrode(s). The dynamic nondimensional field equation of the clamped-clamped microbeam is

$$
w_{\tau \tau}=Q\left(w, w_{\tau}, \tau\right)-R(w)-S\left(w, w_{\tau}\right)
$$

where the time scale is the elastic frequency $\omega_{s}^{2}=E I /\left(\varrho A L^{4}\right)$ and the scaling of the coordinate along the length and the displacement $w$ of the beam are with respect to beam length and gap (distance between beam and electrode), respectively. The restoring force $R$ in (1) is that of a standard EULER-BERNOULLI beam with immovable boundary conditions (b.c.) that includes the effect of residual stresses and nonlinear membrane stiffness, [11].

$$
R(w)=w_{s s s s}-w_{s s}\left[\kappa_{1}+\kappa_{3} \int_{0}^{1} w_{s}^{2} \mathrm{~d} s\right] .
$$

(Subscripts in (1) and (2) denote partial derivatives with respect to scaled time $\tau$ and coordinate $s$ along the length of the beam.) For the sake of clarity, since this paper concentrates on only the static analysis, the author sets aside explicit expressions of time-dependent terms such as the linear and non-linear (squeeze-film) damping, and the actuation term. Such expressions can be found in the literature, e.g. $[3,12,13,14,15,16,17]$. The electrostatic forcing term $Q$ in (1) is inversely proportional to the square of the distance between the structure and electrode(s) [8] assuming that the gap compared to the length of the beam is very small. The distributed force is thus approximated by the expression for a parallel 
capacitor with

$$
\begin{array}{ll}
Q(w)=\frac{\hat{\Gamma} V^{2}}{(1-w)^{2}} & \text { for case I and } \\
Q(w)=\frac{4 \hat{\Gamma} V^{2} w}{\left(1-w^{2}\right)^{2}} & \text { for case II. }
\end{array}
$$

The nondimensional parameters in (2)-(4) are

$$
\begin{array}{ll}
\kappa_{1}=N_{0} L^{2} /(E I), & \kappa_{3}=6(g / H)^{2}, \\
\hat{\Gamma}=6 \varepsilon_{0} L^{4} /\left(E H^{3} g^{3}\right), & V=V_{D C} .
\end{array}
$$

$L, B, H$ are the length, width and thickness of the beam and $A=B H, I=B H^{3} / 12, g$ are the cross section, the second moment of area and the gap between microbeam and electrode(s). $\varepsilon_{0}, E, \rho$, and $N_{0}$ are the dimensional quantities: vacuum permittivity, YounG's modulus, density, and pretensional force, respectively. For a selected set of geometric and material parameters, the constant quantities in (5) take on the numerical values $\kappa_{1}=-24.324$, $\kappa_{3}=6.000$, and $\hat{\Gamma}=0.022$. The nondimensional b.c. of the microbeam are $w(0, \tau)=0$, $w(1, \tau)=0$ and $w_{s}(0, \tau)=0, w_{s}(1, \tau)=0$. A more detailed derivation of the dimensional set of equations of motion are presented in e.g. [3, 4].

\subsection{Formulations of the method of weighted residuals}

In the previous section, the equations of the dynamical system of a doubly-clamped microbeam system under two electrode configurations are presented including nonlinear damping and electrostatic actuation terms. In this section and throughout the rest of the manuscript the emphasis is laid on the static and quasi-static analysis of the microbeam system. 
Thus, the governing equation, deduced from (1), is

$$
\begin{aligned}
R(w)-Q(w) & =0 \quad \text { or } \\
w_{s s s s}-w_{s s}\left[\kappa_{1}+\kappa_{3} \int_{0}^{1} w_{s}^{2} \mathrm{~d} s\right]-Q(w) & =0
\end{aligned}
$$

with $Q(w)$ given in (3) and (4).

The system in (6) is expressed in terms of a nonlinear differential operator $L$ acting on the function $w$ to produce the function $f$

$$
L(w(s))=f(s)
$$

with $L(w(s))=R(w(s))$ and $f(s)=Q(w)$. While some approximation methods begin directly with the strong formulation of the problem (given by (6) or (7) and b.c.), the method of weighted residuals is deduced from the weak formulation of the problem. The weak statement of the problem defined in (7) is

$$
\int_{0}^{1}(L(w)-f) W \mathrm{~d} s=0,
$$

in which $w$ is a trial function (satisfying the geometric b.c.) and $W$ is a weighting function (satisfying the homogeneous counterpart of the geometric b.c.). Both, trial and weighting functions, are arbitrary functions taken from collections or function spaces of trial and weighting solutions, $\mathcal{S}$ and $\mathcal{V}$, that satisfy aforementioned geometric b.c. [18].

For the approximation method the trial and weighting functions are members of finitedimensional function spaces $\mathcal{S}^{h}$ and $\mathcal{V}^{h}$, with $\mathcal{S}^{h} \subset \mathcal{S}$ and $\mathcal{V}^{h} \subset \mathcal{V}$, respectively. The superscript $h$ denotes the characteristic length scale, by which the system is discretized. In the considered problem, $w(s)$ in (7) is approximated by the function $\tilde{w}$, which is a linear 
combination of basis functions

$$
w \cong \tilde{w}=\sum_{m=1}^{M} q_{m} \Phi_{m}
$$

where $q_{m}, \Phi_{m}$, and $M$ are the deflection, the linear undamped modeshapes, and the number of mode shapes used in the approximation, respectively. The linear eigenvalue problem includes terms associated with the axial tension force and the linearized electrostatic actuation force. The shape functions, which satisfy the b.c. exactly [3], are normalized such that $\int_{0}^{1} \Phi_{i} \Phi_{j} \mathrm{~d} s=$ $\delta_{i j}$ and governed by

$$
\Phi_{m}^{i v}-\kappa_{1} \Phi_{m}^{\prime \prime}=\omega_{m}^{2} \Phi_{m}
$$

where $\omega_{m}$ is the $m$ th natural frequency of the microbeam [4]. The associated b.c. are $w(0)=$ $0, w(1)=0$ and $w_{s}(0)=0, w_{s}(1)=0$. The formulation of the MWR is then

$$
\int_{0}^{1} E(w(s)) W_{m}(s) \mathrm{d} s=0 \quad \text { for } \quad m=1,2, \ldots, M,
$$

with $E(w(s))=L(w(s))-f(s)$ and $W_{m}$ being the residual and the weight functions, respectively. The inner product (11) is the approximate (weak) formulation of (6) or (7), i.e. if (11) is satisfied for all $W_{m}$, the differential equation (6) must be satisfied at all points of the domain.

In the GM the weight functions $W_{m}$ are chosen to be the shape or basis functions $W_{m}=$ $\Phi_{m}$. The residual of a continuous function is zero if it is orthogonal to each member of the complete set of basis functions [10], i.e. if the basis functions $\Phi_{m}$ are expressed as members 
of the expansion (9). The GM for cases I and II becomes

$$
\begin{aligned}
& \int_{0}^{1} \Phi_{m}\left[\tilde{w}_{s s s}-\tilde{w}_{s s}\left(\kappa_{1}+\kappa_{3} \int_{0}^{1} \tilde{w}_{s}^{2} \mathrm{~d} s\right)-\frac{\hat{\Gamma} V_{D C}^{2}}{(1-\tilde{w})^{2}}\right] \mathrm{d} s=0 \\
& \int_{0}^{1} \Phi_{m}\left[\tilde{w}_{s s s s}-\tilde{w}_{s s}\left(\kappa_{1}+\kappa_{3} \int_{0}^{1} \tilde{w}_{s}^{2} \mathrm{~d} s\right)-\frac{4 \hat{\Gamma} V_{D C}^{2} \tilde{w}}{\left(1-\tilde{w}^{2}\right)^{2}}\right] \mathrm{d} s=0 \\
& \quad \text { for } m=1,2, \ldots, M .
\end{aligned}
$$

The linearized problems of (12) and (13) are self-adjoint eigenvalue problems, for which two eigenfunctions, corresponding to two different eigenvalues, fulfill the orthogonal relationship [19] and thus force the residual to be zero for the complete set of functions $(M \rightarrow \infty)$. The nonlinear problem quickly converges to a minimum for an increasing number of modeshapes.

The new approach introduced by Younis et al. [4] is, in form, the MWR. The choice of weighting functions $W_{m}=\Phi_{m}(1-\tilde{w})^{2}$ (case I) is generated by the pre-multiplication of the denominator of the forcing term prior to the discretization technique. For case I the formulation of the inner product becomes

$$
\int_{0}^{1} \Phi_{m}(1-\tilde{w})^{2}\left[\tilde{w}_{s s s s}-\tilde{w}_{s s}\left(\kappa_{1}+\kappa_{3} \int_{0}^{1} \tilde{w}_{s}^{2} \mathrm{~d} s\right)-\frac{\hat{\Gamma} V_{D C}^{2}}{(1-\tilde{w})^{2}}\right] \mathrm{d} s=0 .
$$

Although the residual (the terms within the squared brackets of (14)), compared to the previously considered formulation (12), is the same, the weighting functions differ. In specific, the weighting functions have become nonlinear expressions which result in a different "speed" of convergence of the problem. Thus, the number of approximations for satisfying results is different compared to the previous GM. The argumentation that (14) is also a GALERKIN discretization holds only with respect to a redefined residual $E(w(s))$, that is $E(w(s))=$ $(1-\tilde{w})^{2}\left[\tilde{w}_{s s s s}-\tilde{w}_{s s}\left(\kappa_{1}+\kappa_{3} \int_{0}^{1} \tilde{w}_{s}^{2} \mathrm{~d} s\right)-\frac{\hat{\Gamma} V_{D C}^{2}}{(1-\tilde{w})^{2}}\right]$. 
The following section depicts the results of the microbeam for electrode configurations I and II. Throughout the remains of this manuscript the GM mainly refers to results without the pre-multiplication of the denominator of the forcing term $\left(W_{m}=\Phi_{m}\right)$ while the MWR refers to results with having performed the pre-multiplication $\left(W_{m}=\Phi_{m}(1-\tilde{w})^{2}\right)$.

\section{Results for electrode configurations I and II}

In the following the quasi-static displacement DC-voltage curves for the two electrode configurations are shown. A direct comparison between GM and MWR is given by solving the algebraic equations (12), (13) and (14) numerically. The nonlinear algebraic equations obtained from the GM are

$$
\left(\mathbf{K}+\mathbf{K}^{N L}\right) \mathbf{q}-\mathbf{f}=0,
$$

where the elements of the stiffness matrices $\mathbf{K}$ and $\mathbf{K}^{N L}$ are

$$
\begin{gathered}
k_{m m}=\int_{0}^{1}\left[\left(\Phi_{j}^{\prime \prime}\right)^{2}+\kappa_{1}\left(\Phi_{j}^{\prime}\right)^{2}\right] \mathrm{d} s=\omega_{m}^{2}, \\
k_{i m}^{N L}=\kappa_{3} \sum_{k, l}^{1} q_{k} q_{l} \int_{0}^{1} \Phi_{i}^{\prime} \Phi_{m}^{\prime} \mathrm{d} s \int_{0}^{1} \Phi_{k}^{\prime} \Phi_{l}^{\prime} \mathrm{d} s
\end{gathered}
$$

and the vectors $\mathbf{q}=q_{m}$ and $\mathbf{f}=f_{m}$ are the generalized coordinates and actuation force, respectively, with

$$
f_{m}=\int_{0}^{1} \Phi_{m} Q(\tilde{w}) \mathrm{d} s
$$

and $Q(\tilde{w})$ be given by (3) and (4), respectively.

The nonlinear algebraic equations (for case I) obtained from the MWR can be written 
in the following form

$$
\begin{aligned}
& \omega_{m}^{2} q_{m}-2 \sum_{i, j=1}^{M} q_{i} q_{j} \omega_{i}^{2} \int_{0}^{1} \Phi_{i} \Phi_{j} \Phi_{m} \mathrm{~d} s+\sum_{i, j, k=1}^{M} q_{i} q_{j} q_{k} \omega_{i}^{2} \int_{0}^{1} \Phi_{i} \Phi_{j} \Phi_{k} \Phi_{m} \mathrm{~d} s \\
& -\kappa_{3} \sum_{i, j, k=1}^{M} q_{i} q_{j} q_{k} \int_{0}^{1} \Phi_{i}^{\prime} \Phi_{j}^{\prime} \mathrm{d} s \int_{0}^{1} \Phi_{m} \Phi_{k}^{\prime \prime} \mathrm{d} s+2 \kappa_{3} \sum_{i, j, k, l=1}^{1} q_{i} q_{j} q_{k} q_{l} \int_{0}^{1} \Phi_{i}^{\prime} \Phi_{j}^{\prime} \mathrm{d} s \int_{0}^{1} \Phi_{m} \Phi_{k} \Phi_{l}^{\prime \prime} \mathrm{d} s \\
& +\kappa_{3} \sum_{i, j, k, l, n=1}^{M} q_{i} q_{j} q_{k} q_{l} q_{n} \int_{0}^{1} \Phi_{i}^{\prime} \Phi_{j}^{\prime} \mathrm{d} s \int_{0}^{1} \Phi_{m} \Phi_{n} \Phi_{k}^{\prime \prime} \Phi_{l} \mathrm{~d} s-\int_{0}^{1} \hat{\Gamma} V^{2} \Phi_{m} \mathrm{~d} s=0
\end{aligned}
$$

for $m=1,2, \ldots, M$. The expressions of the nonlinear algebraic equations for electrode configuration II are set aside here. They are formulated in analogy to (19) and consequently contain significantly more and also higher-order terms.

\subsection{Case I}

Fig. 2 shows the fixed points of (14), which represents the static equilibrium curve of (1) when having applied the MWR. Slightly different from Younis et al. [4], approximations of only the first three symmetric modes are shown (compare also with Fig. 3 in [4]). The error between the curves in Fig. 2 remains negligibly small for smaller displacements $\left(w_{\max }\right)$ and little over two thirds of the pull-in voltage. For DC-voltages near the pull-in voltage, the displacement curves of the one-, two- and three-mode approximation show significant differences, which Younis et al. reported on in detail. Note, that an increasing number of modes reveals results which converge on the stable lower branch. The convergence on the unstable upper branch is equally achieved for increasing the number of modeshapes, as long as this number is odd. An even number of modeshapes predicts an erroneous form of the unstable upper branch. At this stage, the author has not investigated in this behavior any further. 


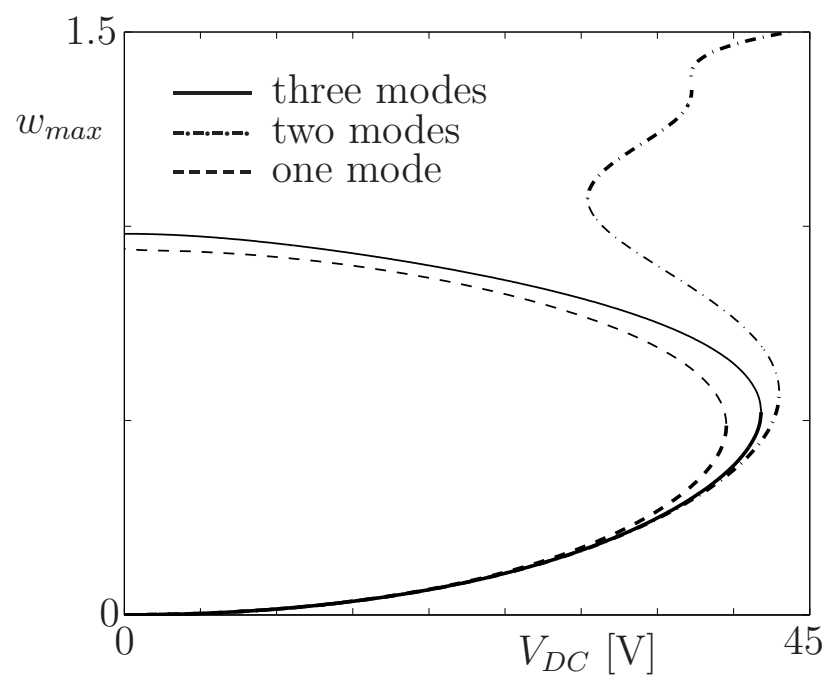

Figure 2: The influence of the number of symmetric modes retained in the MWR on the variation of $w(s)$ with $V_{D C}$; compare also with Fig. 3 in [4]; bold lines: stable, thin lines: unstable.

Fig. 3 portrays the static equilibrium curve of (1) having used the GM (formulation (12)). The error between the curves in Fig. 3 remains negligibly small throughout the shown domain, including the pull-in instability region with large displacements. Fig. 3b) shows the pull-in region zoomed in. Small differences between especially the one- and higher-ordermode approximations are notified here as well. However, the order of magnitude of this error remains small compared to the differences that occur when such curves are compared to equivalent experiments [20]. Fig. 4 shows the comparison between the three-mode MWR and the one-mode GM. Note, that the one-mode GM approximation (12) compared to the three-mode MWR approximation (14) shows no significant difference for the stable branches. Younis et al. reported that a macro model employing the first five symmetric undamped linear modeshapes as base functions in the decomposition technique predicts accurately the static behavior of microbeams [4] p. 676. Furthermore, they show that employing the first 

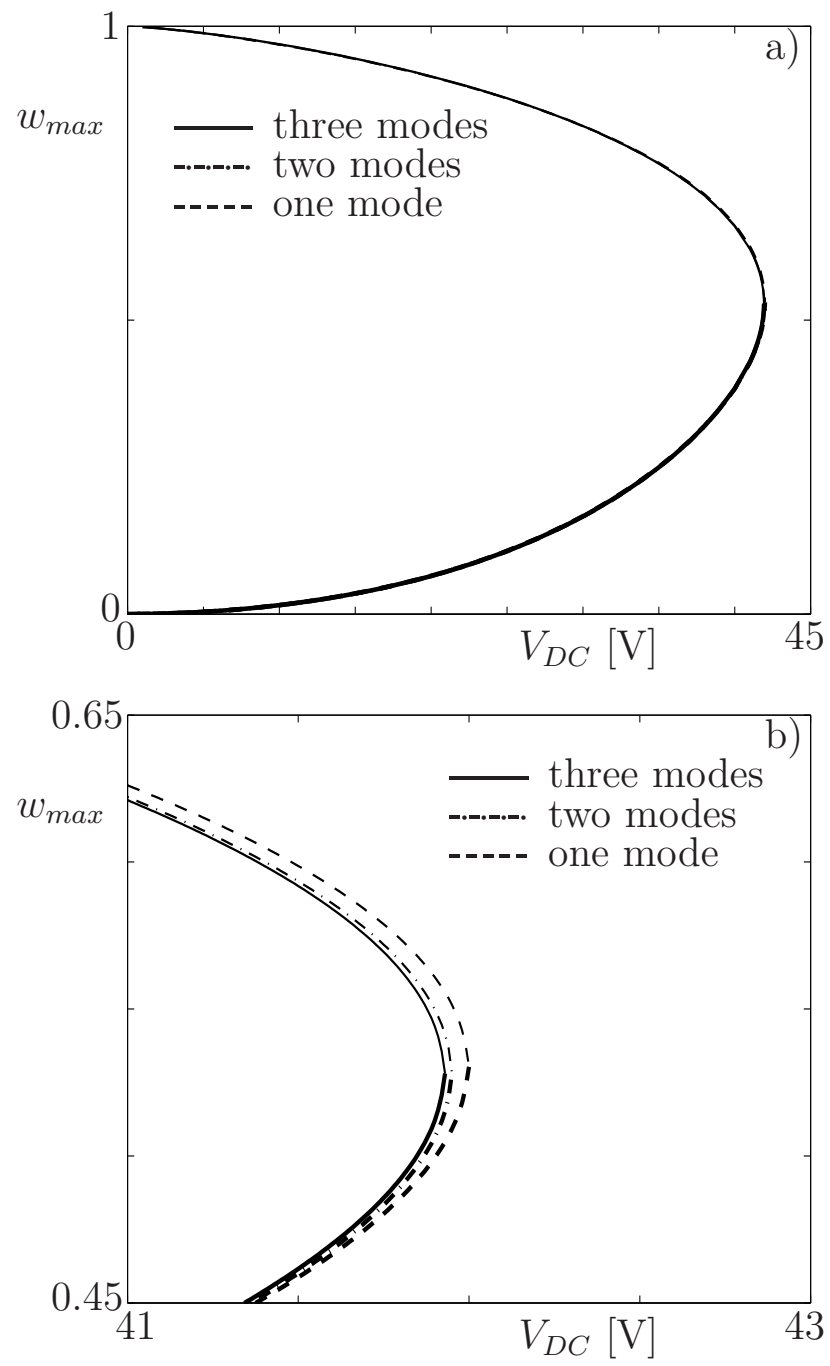

Figure 3: a) The influence of the number of symmetric modes retained in the GM on the variation of $w(s)$ with $V_{D C}$; b) zoom in of a); bold lines: stable, thin lines: unstable.

three symmetric modes already yield sufficient agreement to observation in experiments for the lower (stable) branch until the pull-in instability point.

\subsection{Case II}

Unlike for the non-symmetric electrode configuration, the system's equilibrium points for the double-sided electrode configuration are the trivial solution up to the primary pull-in 


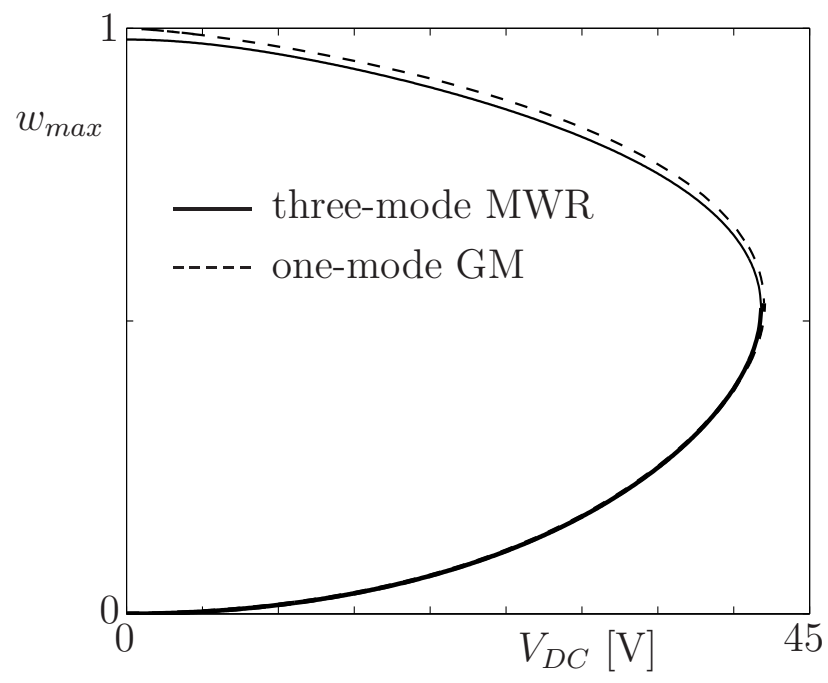

Figure 4: Comparison between the three-mode MWR approximation (14) and the one-mode GM approximation (12); bold lines: stable, thin lines: unstable.

instability of the system. Fig. 5 shows the fixed points of (1) for the decomposition technique with previous multiplication of the denominator of the actuation term

$$
\int_{0}^{1} \Phi_{j}\left(1-\tilde{w}^{2}\right)^{2}\left[\tilde{w}_{s s s s}-\tilde{w}_{s s}\left(\kappa_{1}+\kappa_{3} \int_{0}^{1} \tilde{w}_{s}^{2} \mathrm{~d} s\right)-\frac{4 \hat{\Gamma} V_{D C}^{2} \tilde{w}}{\left(1-\tilde{w}^{2}\right)^{2}}\right] \mathrm{d} s=0 .
$$

As in case I (one-sided electrode), Fig. 5 shows the approximations of employing the single (dashed lines), two (dash-dotted lines), and three (solid lines) symmetric modes. The curves in Fig. 5 are identical (trivial solution) up to the pull-in instability. Beyond the pull-in bifurcation point the error between the three approximations remains small for small displacement equilibria. Near the secondary pull-in instability (see Fig. 5b)), similar differences as seen in case I are noted. The single-mode approximation is underestimating the secondary pull-in point while the two-mode approximation over-computes this point, and the three-mode approximation converges to the minimum value of the residual within negligible error $[4,3]$. 

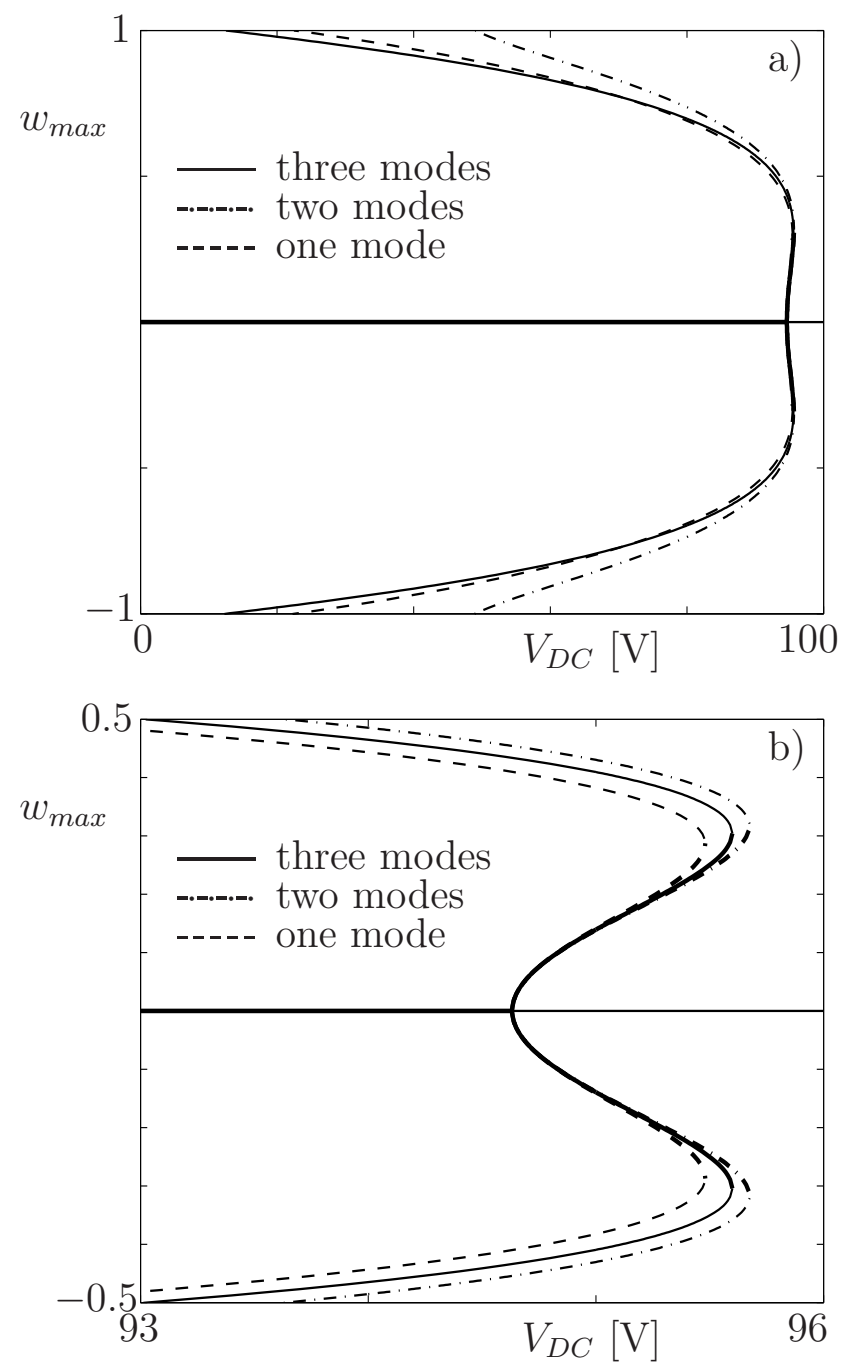

Figure 5: a) The influence of the number of symmetric modes retained in the MWR (20) on the variation of $w(s)$ with $V_{D C}$; b) zoom in of a); bold lines: stable, thin lines: unstable.

Finally, Fig. 6 portrays the static equilibria for the GM. For the trivial solution as well as for the non-trivial branches after the primary pull-in point, the approximations show no significant differences (see also Fig. 6b)). Note the gaps at the primary and secondary pull-in points in Fig. 6b). At these points the numerical computation of the system is facing challenges due to the stiffening character of the actuation integrals (see Eqs. (12) and 

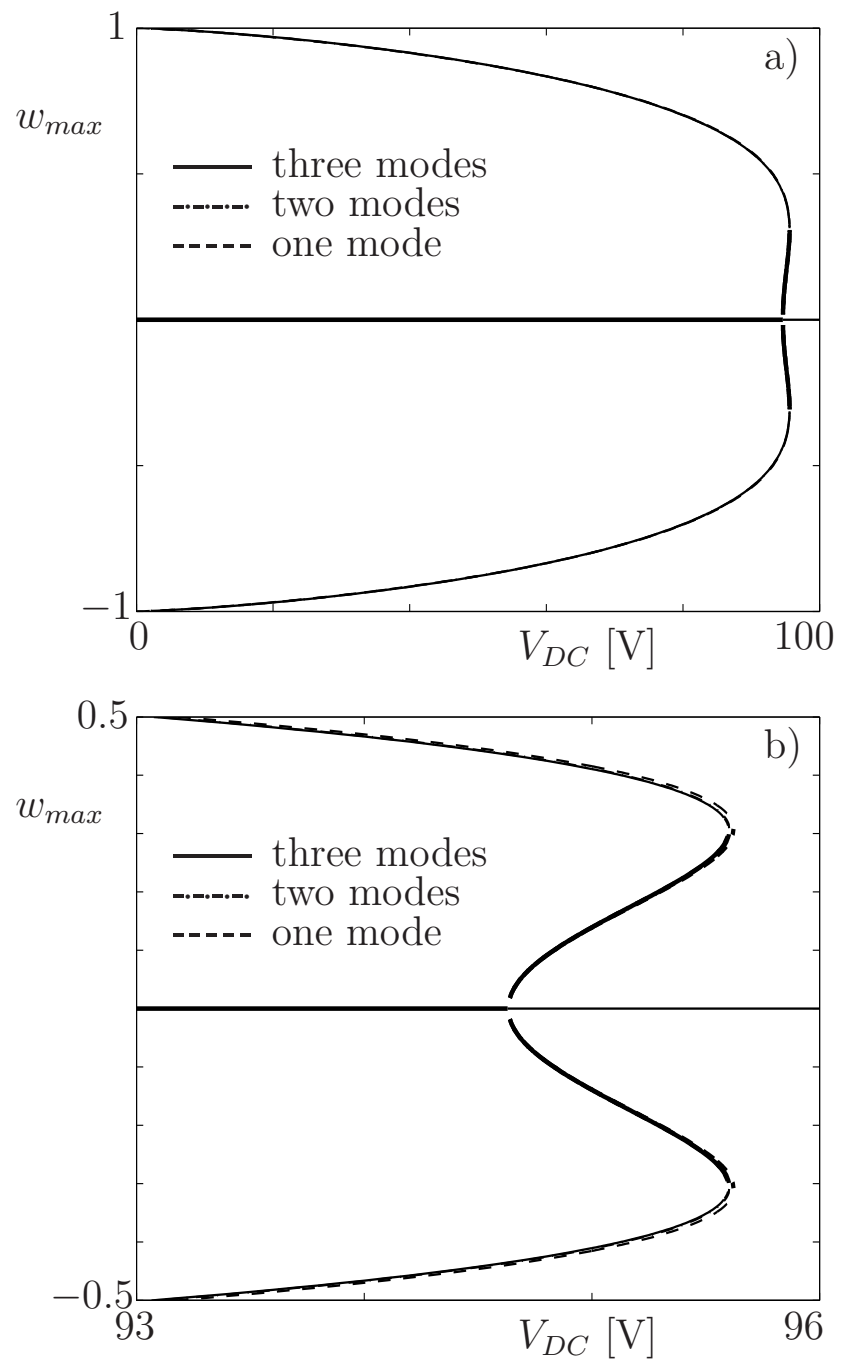

Figure 6: a) The influence of the number of symmetric modes retained in the GM (13) on the variation of $w(s)$ with $V_{D C}$; b) zoom in of a); bold lines: stable, thin lines: unstable.

(13)) at these points. The conclusion which can be drawn from the double-sided electrode configuration is, that if the region of interest is the primary pull-in instability, the single-mode approximation predicts the exact result, regardless the decomposition techniques. (Recall, that this buckling problem is an eigenvalue problem with the first eigenfunction identical to the first shape function.) 


\section{Conclusion}

In this work a comparative study between two different decomposition techniques subject to the modeling of a clamped-clamped microbeam device (for two electrode configurations) is carried out in detail. It is shown that, depending on the system, region of investigation and modeling aims, a first- or higher-order mode approximation predicts accurate results. In the case of the double-sided electrode configuration a single-mode approximation is predicting the primary pull-in instability precisely for both decomposition approaches. This is due to the fact that static equilibria are the trivial solution. The associated linearized eigenvalue problem, which includes the pre-tensional and linearized electrostatic terms, predicts the critical value of the pull-in voltage exactly. For large (nonlinear) displacements a minimum of the first three but no more than the first five symmetric (linear undamped) modes predict the primary pull-in point accurately for the MWR (with pre-multiplication of the denominator of the forcing term) [4]. In contrast, a single-mode approximation is sufficient enough to predict also large displacement equilibria (including pull-in) if the boundary value problem is discretized following the GM (without pre-multiplication of the denominator of the forcing term). The reason for this is related to the change of weighting functions, which add significant higher order terms to the inner product for the MWR (compare e.g. (12) with (14)) and, thus, change the "speed" of convergence while forcing the integral to zero.

The advantages and disadvantages of both discretization methods are defined along with the modeling aims. Younis' et al. new approach (pre-multiplication of the denominator) provides a model for further analytical investigations, allowing (at least theoretically) for formulations of solutions, bifurcation and stability criteria in closed form. The reduced-order 
model which is deduced from the descritization without pre-multiplication of the denominator needs to be solved numerically and thus, can face challenges in finding solutions whenever the system approaches a singularity, like in the cases of primary and secondary pull-in instabilities. However, it was shown that in choosing the latter decomposition technique (GM), the model predicts accurate results with a first-mode approximation only. The existing error is much smaller than any of the remaining uncertainties which the MEMS world is yet challenged by today.

While this study concentrates on the static analysis of a micro-electromechanical beam structure, a future analogous study needs to reveal the answer in the dynamical realms.

\section{Acknowledgment}

The author thanks Olivier Brüls for helpful discussions and proofreading of the manuscript. Soli Deo Gloria.

\section{References}

[1] Rochus, V., Rixen, D. J., and Golinval, J.-C., 2006, "Monolithic modelling of electromechanical coupling in micro-structures," Int. J. Numer. Meth. Engng, 65(4), pp. 461493.

[2] Gabbay, L., Mehner, J., and Senturia, S., 2000, "Computer-aided generation of nonlinear reduced-order dynamic macromodels - i: Non-stress-stiffened case," J. Microelectromech. Syst., 9(2), pp. 262-268. 
[3] Krylov, S., 2007, "Lyapunov exponents as a criterion for the dynamic pull-in instability of electrostatically actuated microstructures," Int. J. of Nonlin. Mech., 42, pp. 626-642.

[4] Younis, M., Abdel-Rahman, E., and Nayfeh, A., 2003, "A reduced-order model for electrically actuated microbeam-based mems," J. Microelectromech. Syst., 12(5), pp. $672-680$.

[5] Hornstein, S. and Gottlieb, O., 2008, "Nonlinear dynamics, stability and control of the scan process in noncontacting atomic force microscopy," Nonlinear Dyn., 54(1-2), pp. $93-122$.

[6] Hung, E. and Senturia, S., 1999, "Generating efficient dynamical models for microelectromechanical systems from a few finite-element simulation runs," J. Microelectromech. Syst., 8(3), pp. 280-289.

[7] Gutschmidt, S. and Gottlieb, O., 2008, "Nonlinear internal resonances of a microbeam array near the pull-in point," In Proc. of 6th EUROMECH Nonlinear Dynamics Conference, St. Petersburg, Russia.

[8] Griffiths, D., 1981, Introduction to Electrodynamics, Prentice-Hall, Englewood Cliffs, NJ.

[9] Nayfeh, A., Younis, M., and Abdel-Rahman, E., 2005, "Reduced-order models for mems applications," Nonlinear Dyn., 41(1-3), pp. 211-236.

[10] Finlayson, B., 1991, The Method of Weighted Residuals and Variational Principles, Academic Press. 
[11] Nayfeh, A., 2000, Nonlinear Interactions, Wiley-Interscience, New York.

[12] Shabana, A., 1991, Theory of Vibration, Springer-Verlag, New York.

[13] Gottlieb, O. and Champneys, A., 2005, "Global bifurcations of nonlinear thermoelastic microbeams subject to electrodynamic actuation," IUTAM Chaotic Dynamics and Control of Systems and Processes in Mechanics, Springer, 122, pp. 117-126.

[14] Senturia, S., 2001, Microsystem Design, Kluwer Academic Publ., Boston.

[15] Krylov, S. and Maimon, R., 2004, "Pull-in dynamics of an elastic beam actuated by continuously distributed electrostatic force," J. Vib. Acoust., 126(3), pp. 332-342.

[16] Osterberg, P. and Senturia, S., 1997, "M-test: A test chip for mems material property measurement using electrostatically actuated test structures," J. Microelectromech. Syst., 6(2), pp. 107-118.

[17] McCarthy, B., Adams, G., McGruer, N., and Potter, D., 2002, "A dynamic model, including contact bounce, of an electrostatically actuated microswitch," J. Microelectromech. Syst., 11(3), pp. 276-283.

[18] Hughes, T., 1987, The Finite Element Method, Linear Static and Dynamic Finite Element Analysis, Prentice-Hall, INC.

[19] Kamke, E., 1948, Differentialgleichungen, Loesungsmethoden und Loesungen: Band 1, Gewoehliche Differentialgleichungen, Chelsea Publishing Company. 
[20] Tilmans, H. and Legtenberg, R., 1994, "Electrostatically driven vacuum-encapsulated polysilicon resonators. 1. design and fabrication," Sensors and Actuators A-Physical, 45(1), pp. 57-66. 


\section{List of Figure Captions}

1. Figure 1: Definition sketch of the clamped-clamped microbeam system for the onesided electrode configuration.

2. Figure 2: The influence of the number of symmetric modes retained in the MWR on the variation of $w(s)$ with $V_{D C}$; compare also with Fig. 3 in [4]; bold lines: stable, thin lines: unstable.

3. Figure 3: a) The influence of the number of symmetric modes retained in the GM on the variation of $w(s)$ with $V_{D C}$; b) zoom in of a); bold lines: stable, thin lines: unstable.

4. Figure 4: Comparison between the three-mode MWR approximation (14) and the one-mode GM approximation (12); bold lines: stable, thin lines: unstable.

5. Figure 5: a) The influence of the number of symmetric modes retained in the MWR (20) on the variation of $w(s)$ with $V_{D C}$; b) zoom in of a); bold lines: stable, thin lines: unstable.

6. Figure 6: a) The influence of the number of symmetric modes retained in the GM (13) on the variation of $w(s)$ with $V_{D C}$; b) zoom in of a); bold lines: stable, thin lines: unstable. 